\title{
How to be Pessimistic: Choquet Risk and Portfolio Optimization
}

\author{
Gilbert W. Bassett, Jr., Roger Koenker, and Gregory Kordas
}

\begin{abstract}
We review some recent developments in the theory of risk assessment. A pessimistic decision theory emerges that replaces the subjective probability assessments of the Savage expected utility criterion with a Choquet expectation that accentuates the likelihood of the least favorable outcomes. We show that pessimistic portfolio optimization may be formulated as an exercise in quantile regression.
\end{abstract}

\section{Introduction}

Many economic decision problems boil down to a choice among competing random variables. In the von Neumann and Morgenstern (1947) and Savage (1954) formalisms an investor comparing two prospects evaluates the expected utilities of their (subjective) returns distributions: a prospect with return distribution $F$ is preferred to another with distribution $G$ provided that,

$$
\int_{-\infty}^{\infty} u(x) d F(x) \geq \int_{-\infty}^{\infty} u(x) d G(x) .
$$

This approach places a heavy burden on the utility function, $u(x)$, to fully reflect investors' attitudes toward risk, and has been called into question by Ellsberg (1961) and many subsequent authors. In reaction to such criticism an alternative formalism, variously called rank-dependent, or non-additive, or Choquet expected utility, has gradually emerged based on work of Quiggin (1981), Schmeidler (1989) Wakker (1989) and others. See Fishburn (1988) and Starmer (2000) for valuable surveys of this work, placing it in the broader context of other alternatives to expected utility theory. The crucial feature of the Choquet approach is that it allows the investor to systematically distort the probability assessments underlying the Savage calculus and thereby reflect more nuanced attitudes toward risk and uncertainty.

Without delving deeply into technical or philosophical details, we will try to provide an elementary exposition of Choquet expected utility and illustrate how it is connected to some recent developments in risk assessment and the measurement

Version March 21, 2002. The authors would like to express their appreciation to Steve Portnoy and Quanshui Zhao for helpful discussions related to this work. 
of inequality. Our primary objective will be to link the rather abstract idea of Choquet risk with a very concrete new approach to portfolio optimization. We will restrict attention to the case of scalar random variables so we may write expected utility as,

$$
E u(X)=\int_{-\infty}^{\infty} u(x) d F(x)=\int_{0}^{1} u\left(F^{-1}(t)\right) d t .
$$

where $F^{-1}(t)=\inf \{x: F(x) \geq t\}$.

Now let $\nu$ be a distribution function on $[0,1]$ and define the Choquet expected utility of $\mathrm{X}$ as,

$$
E_{\nu} u(X)=\int_{0}^{1} u\left(F^{-1}(t)\right) d \nu(t)
$$

Obviously, for $\nu(t)=t$ we have the Savage special case. The distortion $\nu$ reweights the probability assessments according to their rank order in utility. This presumes, of course, that utility is monotone. The family of distortions, $\nu_{\alpha}(t)=\min \{t / \alpha, 1\}$ for $\alpha \in[0,1]$ will play an important role. Focusing for a moment on a single $\nu_{\alpha}$ we have,

$$
E_{\nu_{\alpha}} u(X)=\alpha^{-1} \int_{0}^{\alpha} u\left(F^{-1}(t)\right) d t
$$

and we see that - relative to the Savage computation of expected utility - the probabilities of the $\alpha$ least favorable outcomes are accentuated and the $1-\alpha$ most favorable outcomes are discounted entirely. This may be interpreted as a form of investor pessimism: subjective probabilities are distorted to make the least favorable events appear more likely and the most favorable events less likely. As your gloomy aunt might put it: "Expect the worst, and you won't be disappointed." The crucial feature of the Choquet expectation is that it restricts the distortion to depend only on the rank of the events. Thus, in comparing two prospects that have the same ordering of events, i.e. random variables that differ only by monotonic transformation, we can revert to the Savage comparison. Such prospects are termed comonotone. ${ }^{1}$

We have, at least once heard it objected against the Choquet view of expected utility that the distortions of pessimism, or optimism, can be simply accomodated into Savage's personalistic view of probability. On this point we reserve judgement, but it seems worthwhile to recall that Savage himself did not think so:

I have, at least once heard it objected against the personalistic view of probability that, according to that view, two people might be of different opinions, according as one is pessimistic and the other optimistic. I am not sure what position I would take in abstract discussion of whether that alleged property of personalistic views would be objectionable, but I think it is clear from the

\footnotetext{
${ }^{1}$ More formally, two random variables, $X, Y$ are comonotone, if there exists monotone functions $f, g$ and a random variable $U \sim U[0,1]$ such that $X=f(U)$ and $Y=g(U)$.
} 
formal definition of qualitative probability that the particular personalistic view sponsored here does not leave room for optimism and pessimism, however these traits may be interpreted, to play any role in the person's judgement of probabilities. (Savage(1954, p. 68)

\subsection{Inequality Assessment}

Measurement of inequality is closely related to risk assessment. We can imagine Veblen's Social Engineer trying to compare income distributions behind the Rawlsian veil of ignorance. A classical measure of inequality is the Gini coefficient,

$$
\gamma=1-2 \int_{0}^{1} L(t) d t
$$

where $L(t)$ is the Lorenz function,

$$
L(t)=\int_{0}^{t} F^{-1}(s) d s / \int_{0}^{1} F^{-1}(s) d s
$$

As noted by Gajdos (2002) the Gini coefficient reflects an assumption of linearity in envy in the sense that a Pigou-Dalton transfer between adjacent individuals at the top of the income distribution has the same effect on the Gini as the same transfer between adjacent individuals at the bottom of the distribution. There has been considerable interest in generalized Gini coefficients that reweight the Lorenz curve according to something other than Lebesgue measure, motivated by the idea that transfers at the bottom of the distribution might be considered more significant. Clearly, the Lorenz curve is closely akin to $\nu_{\alpha}$ Choquet expected utility. It is a linear functional of the quantile function, and thus reweighted Gini's are representable as Choquet expectations. See, e.g. Denneberg (1990).

\subsection{Quotidian Risk}

In the everyday drudgery of decision making - should I rob that bank? should I agree to surgery tomorrow? - we are often confronted with complicated problems of risk assessment. In evaluating the risk of medical interventions we find it helpful to consider the Lehmann quantile treatment effect, see Koenker and Geling (2001). Suppose that as a patient facing surgery you are told that in the absence of surgery you face the survival distribution $S_{0}(t)=1-F_{0}(t)$ while if you elect to have the surgery you face $S_{1}(t)=1-F_{1}(t)$. In the absence of any further information, it is reasonable to evaluate the two prospects on the basis of the quantile treatment effect function,

$$
\delta(t)=F_{0}^{-1}(t)-F_{1}^{-1}(t)=S_{1}^{-1}(1-t)-S_{0}^{-1}(1-t) .
$$

Integrating, we obtain,

$$
\mu_{\delta}=\int_{0}^{1} \delta(t) d t
$$



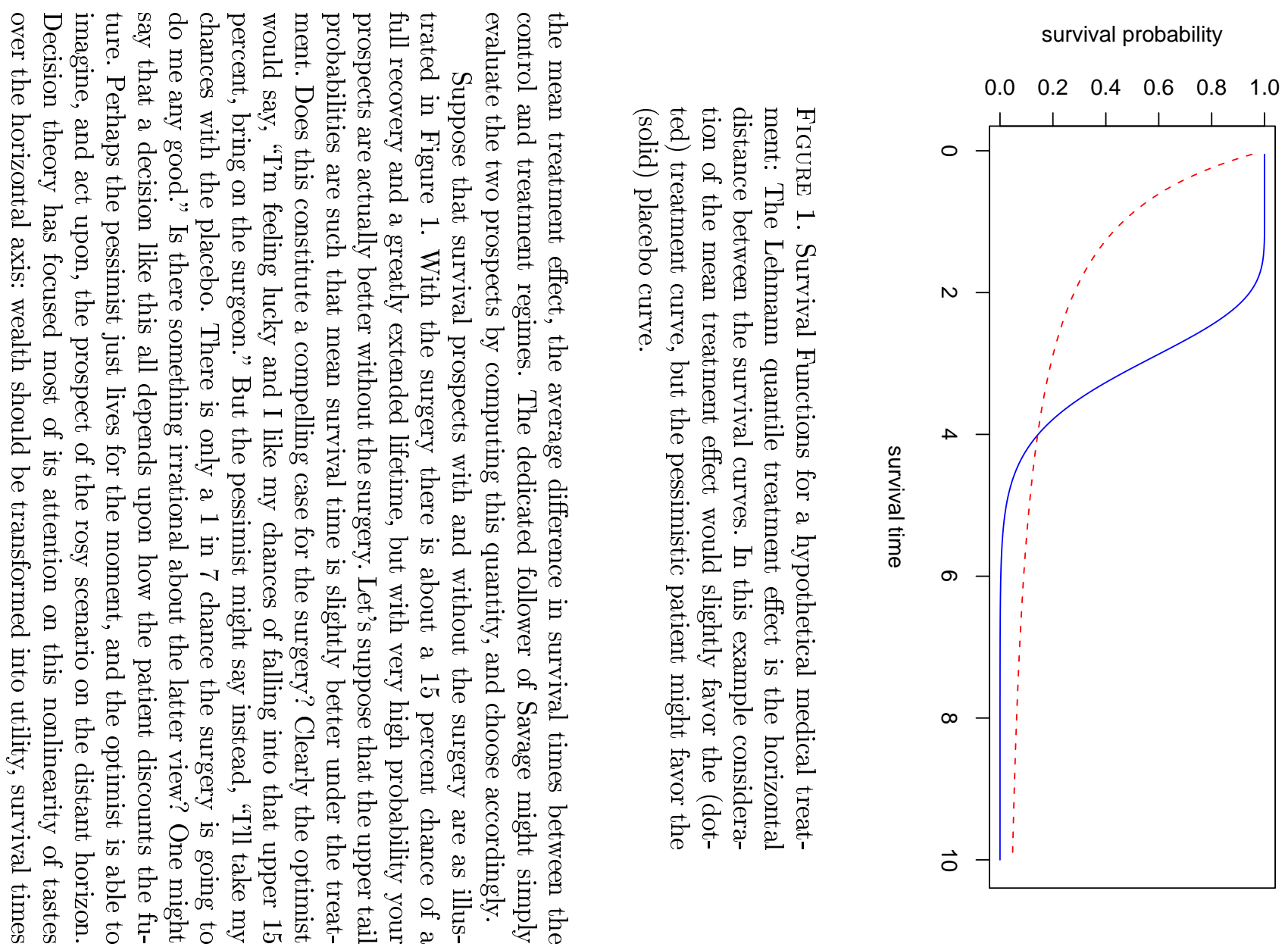
should be discounted. Then we can simply compute expectations. But even after such discounting, we suggest, rational preferences may still admit some element of nonlinearity on the vertical axis of probabilities.

In the next section we provide an overview of some recent work on risk assessment and its relation to Choquet expected utility. The third section links the resulting notion of Choquet risk the optimization problem underlying quantile regression. And it is shown that portfolio optimization methods based on minimizing Choquet risk can be easily implemented using existing algorithms for quantile regression.

\section{Choquet Risk}

In response to regulatory concerns in the finance sector there has been intensive recent interest in the question of how to evaluate portfolio risk. An influential paper in this literature is Artzner, Delbaen, Eber, and Heath (1999), which provides an axiomatic foundation for "coherent" risk measures.

Definition 2.1. (Artzner et al) For real valued random variables $X \in \mathcal{X}$ on $(\Omega, \mathcal{A})$ a mapping $\varrho: \mathcal{X} \rightarrow \mathbb{R}$ is called a coherent risk measure if it is:

1. Monotone: $X, Y \in \mathcal{X}$, with $X \leq Y \Rightarrow \varrho(X) \geq \varrho(Y)$.

2. Subadditive: $X, Y, X+Y \in \mathcal{X}, \Rightarrow \varrho(X+Y) \leq \varrho(X)+\varrho(Y)$.

3. Linearly Homogeneous: For all $\lambda \geq 0$ and $X \in \mathcal{X}, \varrho(\lambda X)=\lambda \varrho(X)$.

4. Translation Invariant: For all $\lambda \in \mathbb{R}$ and $X \in \mathcal{X}, \varrho(\lambda+X)=\varrho(X)-\lambda$.

These requirements rule out many of the conventional measures of risk traditionally used in finance. In particular, measures based on second moments including the standard deviation are ruled out, as are quantile based measures like the value at risk. A measure of risk that has gained considerable recent prominence in the wake of these findings is,

$$
\varrho_{\nu_{\alpha}}(X)=-\alpha^{-1} \int_{0}^{\alpha} F^{-1}(t) d t .
$$

Variants of $\varrho_{\nu_{\alpha}}(X)$ have been suggested under a variety of names: expected shortfall (Acerbi and Tasche (2002)), conditional VaR (Rockafellar and Uryasev (2000)), tail conditional expectation (Artzner, Delbaen, Eber, and Heath (1999))..$^{2}$ For the sake of brevity we will call $\varrho_{\nu_{\alpha}}(X)$ the $\alpha$-risk of the random prospect $X$. Clearly, $\alpha$ risk is simply the negative Choquet $\nu_{\alpha}$ expected return. ${ }^{3}$ Having defined $\alpha$-risk in

${ }^{2}$ The terminology of Uryasev and Rockafellar seems somewhat unfortunate, since it seems to suggest that cVaR is a conditional quantile rather than a conditional mean.

${ }^{3}$ We note in passing, in the hope that it may be deemed relevant at some later point, that elsewhere in the probability literature, e.g. Hurlimann (1998) and Hobson (1998), the random variable $X^{*}$ whose quantile function is,

$$
F_{X^{*}}^{-1}(u)=(1-u)^{-1} \int_{u}^{1} F_{X}^{-1}(v) d v
$$


this way, it is natural to consider the criteria: $\varrho_{\nu_{\alpha}}(X)-\lambda \mu(X)$, or $\mu(X)-\lambda \varrho_{\nu_{\alpha}}(X)$. Minimizing the former criterion may be viewed as minimizing risk subject to a constraint on mean return; maximizing the latter criterion may be viewed as maximizing return subject to a constraint on $\alpha$-risk. Several authors, including Denneberg (1990), Rockafellar and Uryasev (2000), and Jaschke and Küchler (2001), have suggested criteria of this form as alternatives to the classical Markowitz criteria in which $\alpha$-risk is replaced by the standard deviation of the random variable $X$. Since $\mu(X)=\int F_{X}^{-1}(t) d t=-\varrho_{1}(X)$ these criteria are special cases of the following more general class.

Definition 2.2. A risk measure $\varrho$ will be called pessimistic if, for some probability measure $\varphi$ on $[0,1]$,

$$
\varrho(X)=\int_{0}^{1} \varrho_{\nu_{\alpha}}(X) d \varphi(\alpha) .
$$

To see why such risk measures are pessimistic, note that by the Fubini Theorem we can write,

$$
\varrho(X)=-\int_{0}^{1} \alpha^{-1} \int_{0}^{\alpha} F^{-1}(t) d t d \varphi(\alpha)=-\int_{0}^{1} F^{-1}(t) \int_{t}^{1} \alpha^{-1} d \varphi(\alpha) d t .
$$

In the simplest case, we can take $\varphi$ as a sum of point masses, say $d \varphi=\sum_{i} \varphi_{i} \delta_{\tau_{i}}$ with $\varphi_{i}>0$ and $\sum \varphi_{i}=1$ and noting that

$$
\int_{t}^{1} \alpha^{-1} \delta_{\tau}(\alpha) d \alpha=\tau^{-1} I(t<\tau)
$$

we can write

$$
\varrho(X)=\int_{0}^{1} \varrho_{\nu_{\alpha}}(X) d \varphi(\alpha)=-\int_{0}^{1} F^{-1}(t) \gamma(t) d t
$$

where $\gamma(t)=\sum_{i} \varphi_{i} \tau_{i}^{-1} I\left(t<\tau_{i}\right)$. Positivity of the point masses, $\varphi_{i}$, assures that the resulting density weights are decreasing, so the resulting distortion in probabilities acts to accentuate the implicit likelihood of the least favorable outcomes. Such preferences are clearly "pessimistic".

Following Kusuoka (2001) we will impose some additional regularity conditions on $\varrho$ :

Definition 2.3. Let $\mathcal{L}^{\infty}$ denote the space of all bounded real-valued random variables on $(\Omega, \mathcal{F}, \mathbb{P})$ with $\mathbb{P}$ non-atomic. A map $\varrho: \mathcal{L}^{\infty} \rightarrow \mathbb{R}$ is a regular risk measure if:

i. $\varrho$ is law invariant, i.e. $\varrho(X)=\varrho(Y)$ if $X, Y \in \mathcal{L}^{\infty}$ have the same probability law.

ii. $\varrho$ satisfies the Fatou property, i.e. if $\left\{X_{n}\right\}_{i=1}^{n} \subset \mathcal{L}^{\infty}$ are uniformly bounded and converge to $X$ in probability then $\varrho(X)=\liminf _{n \rightarrow \infty} \varrho\left(X_{n}\right)$.

is called the Hardy-Littlewood transform of $X$. Obviously, the $\alpha$-risks constitute the negative Hardy-Littlewood transform of the random variable $-X$. 
iii. $\varrho$ is comonotone, i.e. $X, Y \in \mathcal{L}^{\infty}$ comonotone implies that $\varrho(X+Y)=$ $\varrho(X)+\varrho(Y)$.

The first two regularity conditions impose a relatively weak form of continuity, while the third condition refines slightly the the subadditivity property. We can now succinctly reformulate the main representation result of Kusuoka (2001).

Theorem 2.4. A regular risk measure is coherent if and only if it is pessimistic.

One could, of course, also consider a more general class of Choquet risk measures,

$$
\varrho(X)=-\int F_{X}^{-1}(t) d \nu(t)
$$

for distribution functions $\nu$ on $[0,1]$. Pessimistic risk measures correspond to concave $\nu$, assigning decreasing density on the interval $[0,1]$. "Optimistic" risk measure would have convex $\nu$, thus reweighting more favorable outcomes more heavily, and discounting the likelihood of less favorable eventualities. It is quite plausible to consider $\nu$ that are concave in the lower tail and convex in the upper tail as a way to rationalize the commonly observed willingness to purchase insurance and buy lottery tickets. Quiggin (1993) provides an excellent discussion of possible motivations for these possibilities, so we will resist the temptation to delve further into them. Instead, we now turn to a description of an empirical approach to portfolio optimization based on pessimistic risk measures.

\section{How to be Pessimistic}

Empirical strategies for optimizing $\alpha$-risk lead immediately into the realm of quantile regression. Let $\rho_{\tau}(u)=u(\tau-I(u<0))$ denote the "check function" of Koenker and Bassett (1978) and consider the problem,

$$
\min _{\xi \in \mathbb{R}} E \rho_{\alpha}(X-\xi) \text {. }
$$

We know that any $\xi$ solving this problem is an $\alpha$ th quantile of the random variable $X$. Evaluating at the minimizer, $\xi_{\alpha}$ we find that minimizing the usual $\alpha$-quantile objective function is equivalent to evaluating the sum of expected return and the (Choquet) $\alpha$-risk of $X$, and then multiplying by $\alpha$.

Theorem 3.1. Let $X$ be a real-valued random variable with $E X=\mu<\infty$, then

$$
\min _{\xi \in \mathbb{R}} E \rho_{\alpha}(X-\xi)=\alpha\left(\mu+\varrho_{\nu_{\alpha}}(X)\right) .
$$

Proof. Noting that,

$$
E \rho_{\alpha}(X-\xi)=\alpha(\mu-\xi)-\int_{-\infty}^{\xi}(x-\xi) d F_{X}(x),
$$

is minimized when $\xi_{\alpha}=F_{X}^{-1}(\alpha)$, we have,

$$
E \rho_{\alpha}\left(X-\xi_{\alpha}\right)=\alpha \mu+\alpha \varrho_{\nu_{\alpha}}(X) \text {. }
$$


The empirical analogue of $\alpha$-risk can thus be formulated as

$$
\hat{\varrho}_{\nu_{\alpha}}(x)=(n \alpha)^{-1} \min _{\xi \in \mathbb{R}} \sum_{i=1}^{n} \rho_{\alpha}\left(x_{i}-\xi\right)-\hat{\mu}_{n}
$$

where $\left\{x_{i}: i=1, \ldots, n\right\}$ constitutes a random sample on $X$, and $\hat{\mu}_{n}$ denotes an estimator of $E X=\mu$, presumably, $\bar{x}_{n}$. Of course $\hat{\varrho}_{\nu_{\alpha}}(x)$ could easily be defined in a seemingly more direct manner, but the value of the proposed optimization formulation becomes apparent as soon as we begin to consider portfolios of assets. Let $Y=X^{\top} \pi$ denote a portfolio of assets comprised of $X=\left(X_{1}, \ldots, X_{p}\right)^{\top}$ with portfolio weights $\pi$. Suppose we observe a random sample $\left\{x_{i}=\left(x_{i 1}, \ldots, x_{i p}\right): i=\right.$ $1, \ldots, n\}$ and we wish to consider portfolios minimizing

$$
\min _{\pi} \varrho_{\nu_{\alpha}}(Y)-\lambda \mu(Y)
$$

This is evidently equivalent to simply minimizing $\varrho_{\nu_{\alpha}}(Y)$ subject to a constraint on mean return. We will impose the additional constraint that the portfolio weights $\pi$ sum to one, and reformulate the problem as,

$$
\min _{\pi} \varrho_{\nu_{\alpha}}\left(X^{\top} \pi\right) \text { s.t. } \mu\left(X^{\top} \pi\right)=\mu_{0}, 1^{\top} \pi=1 .
$$

Taking the first asset as numeraire we can write the sample analogue of this problem as

$$
\min _{(\beta, \xi) \in \mathbb{R}^{p}} \sum_{i=1}^{n} \rho_{\alpha}\left(x_{i 1}-\sum_{j=2}^{p}\left(x_{i 1}-x_{i j}\right) \beta_{j}-\xi\right) \text { s.t. } \bar{x}^{\top} \pi(\beta)=\mu_{0},
$$

where $\pi(\beta)=\left(1-\sum_{j=2}^{p} \beta_{j}, \beta^{\top}\right)^{\top}$. It is easy to verify that the solution is invariant to the choice of the numeraire asset. At the solution, $\hat{\xi}$ is the $\alpha$ th sample quantile of the chosen portfolio's returns distribution. The required return constraint implicitly corresponds to a particular $\lambda$ in the original specification (3.1). Note that we have not (yet) imposed any further constraints on the portfolio weights $\beta$, but given the linear programming form of the problem (3.2) it would be straightforward to do so.

The problem posed in (3.2) is (almost) a conventional quantile regression problem. The only idiosyncrasy is the mean return constraint, but it is easy to impose this constraint by simply adding a single pseudo observation to the sample consisting of response $\kappa\left(\bar{x}_{i}-\mu_{0}\right)$ and design row $\kappa\left(0, \bar{x}_{1}-\bar{x}_{2}, \ldots, \bar{x}_{1}-\bar{x}_{p}\right)^{\top}$. For sufficiently large $\kappa$ we are assured that the constraint will be satisfied. Varying $\mu_{0}$ we obtain an empirical $\alpha$-risk frontier.

Example 1. Our first illustration of the approach will be based on a small artificial dataset. We generate independent returns on 4 assets with marginal densities illustrated in Figure 1. Solving (3.2) with various values of $\mu_{0}$, we obtain portfolios with weights illustrated in Figure 1c and the risk-return frontier appearing in Figure 2. How do these portfolios compare to the mean-variance portfolios of the classical theory for this data? In Figure 3 we compare the returns densities of 


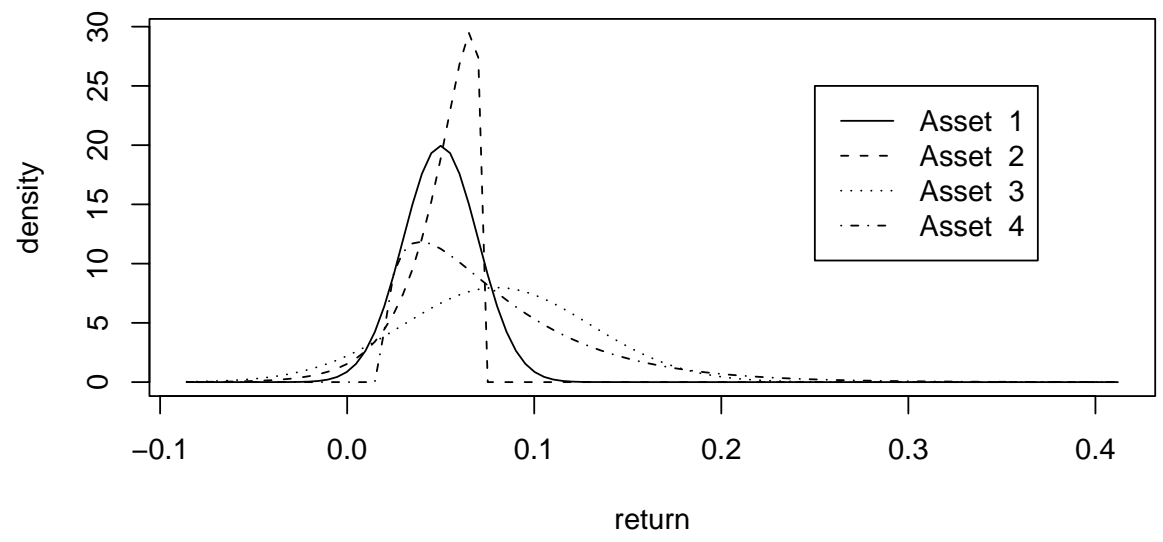

Figure 2. Four Asset Densities for Example 1: We will construct portfolios comprised of four independent asset returns with marginal densities illustrated above.
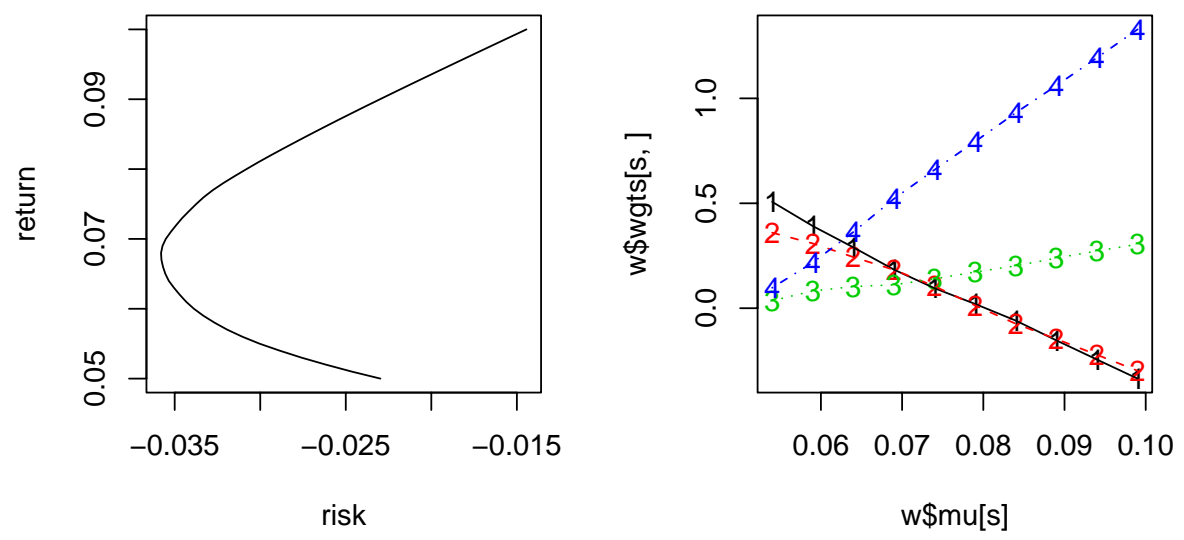

FiguRE 3. The $\alpha$-risk-return frontier: For the four artificial asset returns series we compute the risk-return frontier according to (3.2) in panel b. The portfolio weights as functions of the required mean return are depicted in Panel c. 


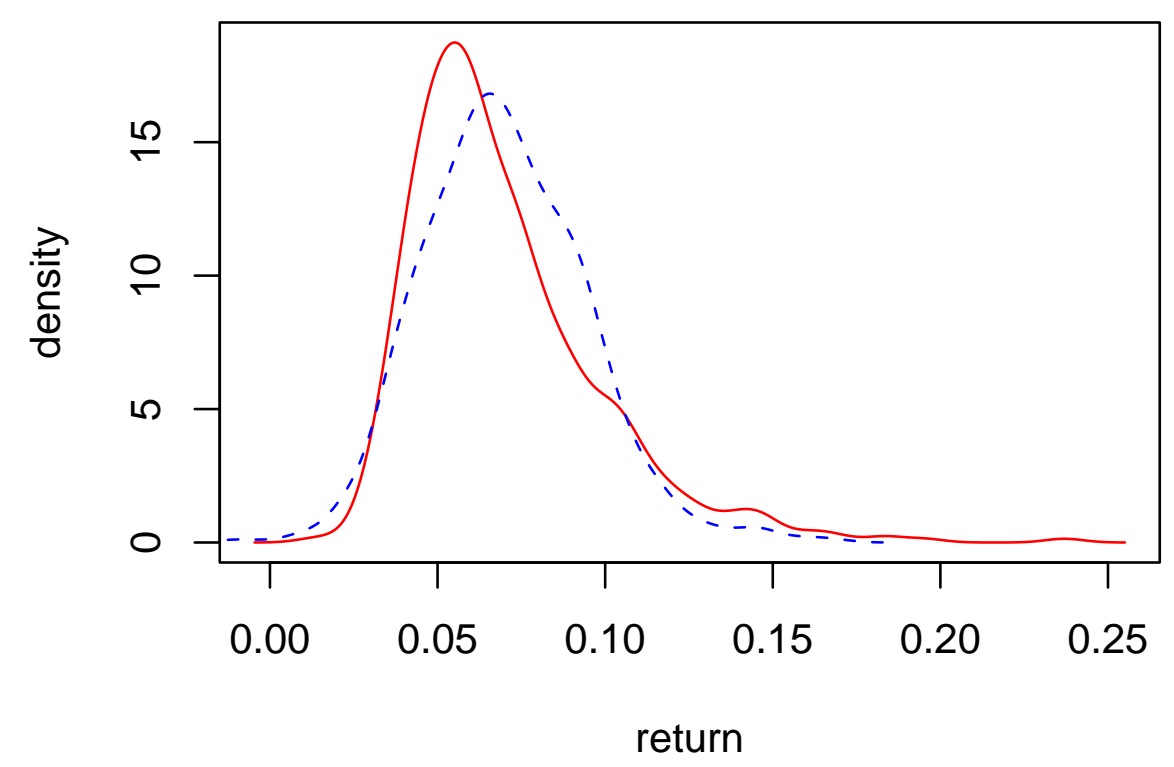

Figure 4. Mean-variance vs. $\alpha$-risk portfolio returns: Based on the data of example 1 we illustrate the estimated density of portfolio returns for the optimal mean-variance portfolio and the optimal $\alpha$-risk portfolio for required mean return of .07. Note the the solid curve representing the $\alpha$-risk returns has better performance than the dotted mean-variance density in both tails.

the optimal $\alpha$-risk portfolio with the optimal mean variance portfolio performance for two different levels of required mean return. The four assets should be seen as two pairs: a lower pair consisting of a normal density with mean .04 and standard deviation .02 and a left skewed reversed $\chi_{3}^{2}$ density with the same mean and standard deviation, and an upper pair consisting of a normal density with mean return .08 and standard deviation .05 , and a right skewed $\chi_{3}^{2}$ density with the the same mean and standard deviation. The $\alpha$-risk portfolios tend to prefer the right skewed asset and disdain the left skewed one. For example at required mean return .07 , the $\alpha$-risk portfolio puts weight .55 on right skewed asset and only .11 weight on its normal counterpart, while the mean-variance portfolio places equal weight, .33 , on both. 
Although the $\alpha$-risks provide a convenient one-parameter family of coherent risk measures, they are obviously rather simplistic. As we have already suggested, it is natural to consider weighted averages of $\alpha$-risks:

$$
\varrho_{\nu}(X)=\sum_{k=1}^{m} \nu_{k} \varrho_{\nu_{\alpha_{k}}}(X) \text {. }
$$

Where the weights, $\nu_{k}: k=1, \ldots, m$, are positive and sum to one. This risk criterion can also be easily implemented empirically extending the formulation in

$$
\min _{(\beta, \xi) \in \mathbb{R}^{p+m}} \sum_{k=1}^{m} \sum_{i=1}^{n} \nu_{k} \rho_{\alpha}\left(x_{i 1}-\sum_{j=2}^{p}\left(x_{i 1}-x_{i j}\right) \beta_{j}-\xi_{k}\right) \text { s.t. } \bar{x}^{\top} \pi(\beta)=\mu_{0} .
$$

The only new wrinkle is the appearance of $m$ distinct intercept parameters representing the $m$ estimated quantiles of the returns distribution of the chosen portfolio. In effect we have simply stacked $m$ distinct quantile regression problems on top of one another and introduced a distinct intercept parameter for each of them, while constraining the portfolio weights to be the same for each quantile. Since the $\nu_{k}$ are all positive, they may passed inside the $\rho_{\alpha}$ function to rescale the argument. The statistical theory of such constrained quantile regression estimators is discussed in Koenker (1984).

\section{Extensions}

There are many loose ends and topics for future research. An important byproduct of the quantile regression formulation of the $\alpha$-risk portfolio optimization problem is the attendant statistical inference provided. This is most straightforward in the case of the simple $\alpha$-risk objective function, but can be extended to the general case of weighted sums of $\alpha$-risks. We hope to consider these issues in future work. There are also many other possible refinements including the incorporation of additional constraints. Upper and lower bounds on the positions held in the portfolio would often be appropriate, and would be easy to implement, as would shrinkage of portfolio weights toward some a priori portfolio. Most importantly, it is necessary to explore, prod, and test the Choquet approach on realistic applied problems.

The expected utility theory of von Neumann and Morgenstern is firmly embedded in the zeitgeist of modern decision theory. It has withstood more than a half century of severe criticism, whether a viable alternative theory can be built on the foundations of Choquet expectation remains an open question. But it is a question that deserves further investigation.

\section{References}

ACERBI, C., AND D. TASCHE (2002): "Expected Shortfall: A Natural coherent alternative to Value at Risk," Economic Notes. 
Artzner, P., F. Delbaen, J.-M. Eber, and D. Heath (1999): "Coherent Measures of Risk," Math. Finance, 9, 203-228.

Denneberg, D. (1990): "Premium Calculation: Why standard deviation should be replaced by absolute deviation," ASTIN Bulletin, 20, 181-190.

EllsberG, D. (1961): "Risk, Ambiguity and Savage Axioms," Quarterly J. Econ., 75, 643-669.

Fishburn, P. (1988): Nonlinear Preference and Utility Theory. Johns Hopkins Press.

Gajdos, T. (2002): "Measuring Inequalities without Linearity in Envy: Choquet Integrals for Symmetric Capacities," J. Econ. Theory, forthcoming.

Hobson, D. G. (1998): "Robust Hedging of the Lookback Option," Finance and Stochastics, 2, 329-347.

Hurlimann, W. (1998): "On Stop-Loss Order and the Distortion Pricing Principle," ASTIN Bulletin, 28, 119-134.

Jaschke, S., AND U. KüChler (2001): "Coherent Risk Measure and Good Deal Bounds," Finance and Stochastics, 5, 181-200.

Koenker, R. (1984): "A Note on L-estimators for Linear Models," Stat and Prob Letters, $2,323-325$.

Koenker, R., And G. Bassett (1978): "Regression Quantiles," Econometrica, 46, 3350 .

Koenker, R., And O. Geling (2001): "Reappraising Medfly Longevity: A quantile regression survival analysis," J. of Am. Stat. Assoc., 96, 458-468.

Kusuoka, S. (2001): "On Law Invariant Coherent Risk Measures," Advances in Math. Econ., 3, 83-95.

Quiggin, J. (1981): "Risk Perception and Risk Aversion among Australian Farmers," Aust. J. of Ag. Econ., 25, 160-169.

(1993): Generalized Expected Utility Theory. Kluwer Academic Publishers.

Rockafellar, R., AND S. URYasev (2000): "Optimization of conditional VaR," J. of Risk, 2, 21-41.

SaVAGE, L. (1954): Foundations of Statistics. Wiley.

SchmeIDleR, D. (1989): "Subjective Probability and Expected Utility without Additivity," Econometrica, 57, 571-587.

Starmer, C. (2000): "Developments in Non-expected Utility Theory: The Hunt for a Descriptive Theory of Choice under Risk," J. of Economic Literature, 38, 332-382.

von Neumann, J., And O. Morgenstern (1947): Theory of Games and Economic Behavior. Princeton.

Wakker, P. (1989): Additive Representations of Preferences: A New Foundation of Decision Analysis. Kluwer Academic Publishers.

University of Illinois at Chicago

University of Illinois at Urbana-Champaign

University of Pennsylvania 